\title{
Two-parameter Srivastava polynomials and several series identities
}

\author{
Cem Kaanoglu ${ }^{1 *}$ and Mehmet Ali Özarslan ${ }^{2}$
}

\section{"Correspondence:}

kaanoglu@ciu.edu.tr

1'Faculty of Engineering, Cyprus

International University, Mersin 10,

Nicosia, Turkey

Full list of author information is

available at the end of the article

\begin{abstract}
In the present paper, we introduce two-parameter Srivastava polynomials in one, two and three variables by inserting new indices, where in the special cases they reduce to (among others) Laguerre, Jacobi, Bessel and Lagrange polynomials. These polynomials include the family of polynomials which were introduced and/or investigated in (Srivastava in Indian J. Math. 14:1-6, 1972; González et al. in Math. Comput. Model. 34:133-175, 2001; Altın et al. in Integral Transforms Spec. Funct. 17(5):315-320, 2006; Srivastava et al. in Integral Transforms Spec. Funct. 21(12):885-896, 2010; Kaanoglu and Özarslan in Math. Comput. Model. 54:625-631, 2011). We prove several two-sided linear generating relations and obtain various series identities for these polynomials. Furthermore, we exhibit some illustrative consequences of the main results for some well-known special polynomials which are contained by the two-parameter Srivastava polynomials.

MSC: $33 C 45$
\end{abstract}

\section{Introduction}

Let $\left\{A_{n, k}\right\}_{n, k=0}^{\infty}$ be a bounded double sequence of real or complex numbers, let $[a]$ denote the greatest integer in $a \in \mathbb{R}$, and let $(\lambda)_{v},(\lambda)_{0} \equiv 1$, denote the Pochhammer symbol defined by

$$
(\lambda)_{v}:=\frac{\Gamma(\lambda+v)}{\Gamma(\lambda)}
$$

by means of familiar gamma functions. In 1972, Srivastava [1] introduced the following family of polynomials:

$$
S_{n}^{N}(z):=\sum_{k=0}^{\left[{ }_{N}^{n}\right]} \frac{(-n)_{N k}}{k !} A_{n, k} z^{k} \quad\left(n \in \mathbb{N}_{0}=\mathbb{N} \cup\{0\} ; N \in \mathbb{N}\right),
$$

where $\mathbb{N}$ is the set of positive integers.

Afterward, González et al. [2] extended the Srivastava polynomials $S_{n}^{N}(z)$ as follows:

$$
S_{n, m}^{N}(z):=\sum_{k=0}^{\left[\frac{n}{N}\right]} \frac{(-n)_{N k}}{k !} A_{n+m, k} z^{k} \quad\left(m, n \in \mathbb{N}_{0} ; N \in \mathbb{N}\right)
$$

@ 2013 Kaanoglu and Özarslan; licensee Springer. This is an Open Access article distributed under the terms of the Creative Commons Attribution License (http://creativecommons.org/licenses/by/2.0), which permits unrestricted use, distribution, and reproduction in any medium, provided the original work is properly cited. 
and investigated their properties extensively. Motivated essentially by the definitions (1) and (2), scientists investigated and studied various classes of Srivastava polynomials in one and more variables.

In [3], the following family of bivariate polynomials was introduced:

$$
S_{n}^{m, N}(x, y):=\sum_{k=0}^{\left[\frac{n}{N}\right]} A_{m+n, k} \frac{x^{n-N k}}{(n-N k) !} \frac{y^{k}}{k !} \quad\left(n, m \in \mathbb{N}_{0}, N \in \mathbb{N}\right),
$$

and it was shown that the polynomials $S_{n}^{m, N}(x, y)$ include many well-known polynomials such as Lagrange-Hermite polynomials, Lagrange polynomials and Hermite-Kampé de Feriét polynomials.

In [4], Srivastava et al. introduced the three-variable polynomials

$$
\begin{aligned}
& S_{n}^{m, M, N}(x, y, z) \\
& \quad:=\sum_{k=0}^{\left[\frac{n}{N}\right]\left[\frac{k}{M}\right]} \sum_{l=0}^{A_{m+n, k, l}} \frac{x^{l}}{l !} \frac{y^{k-M l}}{(k-M l) !} \frac{z^{n-N k}}{(n-N k) !} \quad\left(m, n \in \mathbb{N}_{0} ; M, N \in \mathbb{N}\right),
\end{aligned}
$$

where $\left\{A_{m, n, k}\right\}$ is a triple sequence of complex numbers. Suitable choices of $\left\{A_{m, n, k}\right\}$ in equation (3) give a three-variable version of well-known polynomials (see also [5]). Recently, in [6], the multivariable extension of the Srivastava polynomials in $r$-variable was introduced

$$
\begin{aligned}
& S_{n}^{m, N_{1}, N_{2}, \ldots, N_{r-1}}\left(x_{1}, x_{2}, \ldots, x_{r}\right)
\end{aligned}
$$

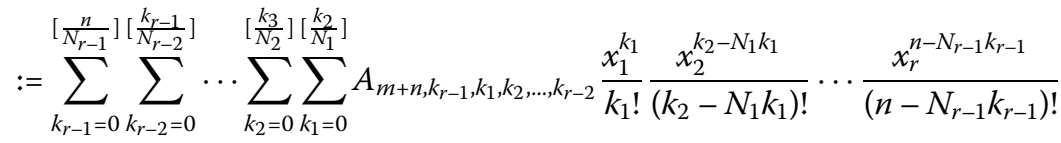

$$
\begin{aligned}
& \left(m, n \in \mathbb{N}_{0} ; N_{1}, N_{2}, \ldots, N_{r-1} \in \mathbb{N}\right) \text {, }
\end{aligned}
$$

where $\left\{A_{m, k_{r-1}, k_{1}, k_{2}, \ldots, k_{r-2}}\right\}$ is a sequence of complex numbers.

In this paper we introduce the two-parameter Srivastava polynomials in one and more variables by inserting new indices. These polynomials include the family of polynomials which were introduced and/or investigated in [1-4, 6, 7] and [8]. We prove several twosided linear generating relations and obtain various series identities for these polynomials. Furthermore, we exhibit some illustrative consequences of the main results for some wellknown special polynomials which are contained by the two-parameter Srivastava polynomials.

\section{Two-parameter one-variable Srivastava polynomials}

In this section we introduce the following family of two-parameter one-variable polynomials:

$$
S_{n}^{m_{1}, m_{2}}(x):=\sum_{k=0}^{n} \frac{(-n)_{k}}{k !} A_{m_{1}+m_{2}+n, m_{2}+k} x^{k} \quad\left(m_{1}, m_{2}, n, k \in \mathbb{N}_{0}\right),
$$


where $\left\{A_{n, k}\right\}$ is a bounded double sequence of real or complex numbers. Note that appropriate choices of the sequence $A_{n, k}$ give one-variable versions of the well-known polynomials.

Remark 2.1 Choosing $A_{m, n}=(-\alpha-m)_{n}\left(m, n \in \mathbb{N}_{0}\right)$ in (5), we get

$$
S_{n}^{m_{1}, m_{2}}\left(\frac{-1}{x}\right)=(-1)^{m_{2}}\left(\alpha+m_{1}+n+1\right)_{m_{2}} \frac{n !}{(-x)^{n}} L_{n}^{\left(\alpha+m_{1}\right)}(x),
$$

where $L_{n}^{(\alpha)}(x)$ are the classical Laguerre polynomials given by

$$
L_{n}^{(\alpha)}(x)=\frac{(-x)^{n}}{n !} F_{2}\left(-n,-\alpha-n ;-; \frac{-1}{x}\right) .
$$

Remark 2.2 Setting

$$
A_{m, n}=\frac{(\alpha+\beta+1)_{2 m}(-\beta-m)_{n}}{(\alpha+\beta+1)_{m}(-\alpha-\beta-2 m)_{n}} \quad\left(m, n \in \mathbb{N}_{0}\right)
$$

in (5), we obtain

$$
\begin{aligned}
S_{n}^{m_{1}, m_{2}}\left(\frac{2}{1+x}\right) \\
=\frac{(\alpha+\beta+1)_{2 m_{1}+2 m_{2}+2 n}\left(-\beta-m_{1}-m_{2}-n\right)_{m_{2}}\left(1+\alpha+\beta+2 m_{1}+m_{2}\right)_{n}}{(\alpha+\beta+1)_{m_{1}+m_{2}+n}\left(-\alpha-\beta-2 m_{1}-2 m_{2}-2 n\right)_{m_{2}}\left(1+\alpha+\beta+2 m_{1}+m_{2}\right)_{2 n}} \\
\quad \times n !\left(\frac{2}{1+x}\right)^{n} P_{n}^{\left(\alpha+m_{1}+m_{2}, \beta+m_{1}\right)}(x),
\end{aligned}
$$

where $P_{n}^{(\alpha, \beta)}(x)$ are the classical Jacobi polynomials.

Remark 2.3 If we set $A_{m, n}=(\alpha+m-1)_{n}\left(m, n \in \mathbb{N}_{0}\right)$ in (5), then we get

$$
S_{n}^{m_{1}, m_{2}}\left(\frac{-x}{\beta}\right)=\left(\alpha+m_{1}+m_{2}+n-1\right)_{m_{2}} y_{n}\left(x, \alpha+m_{1}+2 m_{2}, \beta\right) \quad(\beta \neq 0),
$$

where $y_{n}(x, \alpha, \beta)$ are the classical Bessel polynomials given by

$$
y_{n}(x, \alpha, \beta)={ }_{2} F_{0}\left(-n, \alpha+n-1 ;-; \frac{-x}{\beta}\right) .
$$

Theorem 2.4 Let $\{f(n)\}_{n=0}^{\infty}$ be a bounded sequence of complex numbers. Then

$$
\begin{aligned}
& \sum_{m_{1}, m_{2}, n=0}^{\infty} f\left(n+m_{1}+m_{2}\right) S_{n}^{m_{1}, m_{2}}(x) \frac{w_{1}^{m_{1}}}{m_{1} !} \frac{w_{2}^{m_{2}}}{m_{2} !} \frac{t^{n}}{n !} \\
& =\sum_{m_{1}, m_{2}=0}^{\infty} f\left(m_{1}+m_{2}\right) A_{m_{1}+m_{2}, m_{2}} \frac{\left(w_{1}+t\right)^{m_{1}}}{m_{1} !} \frac{\left(w_{2}+(-x t)\right)^{m_{2}}}{m_{2} !}
\end{aligned}
$$

provided each member of the series identity (6) exists. 
Proof Let the left-hand side of (6) be denoted by $\Psi(x)$. Then, using the definition of $S_{n}^{m_{1}, m_{2}}(x)$ on the left-hand side of (6), we have

$$
\begin{aligned}
\Psi(x) & =\sum_{m_{1}, m_{2}, n=0}^{\infty} f\left(n+m_{1}+m_{2}\right) \sum_{k=0}^{n} \frac{(-n)_{k}}{k !} A_{m_{1}+m_{2}+n, m_{2}+k} x^{k} \frac{w_{1}^{m_{1}}}{m_{1} !} \frac{w_{2}^{m_{2}}}{m_{2} !} \frac{t^{n}}{n !} \\
& =\sum_{m_{1}, m_{2}, n=0}^{\infty} f\left(n+m_{1}+m_{2}\right) \sum_{k=0}^{n} \frac{1}{k !} A_{m_{1}+m_{2}+n, m_{2}+k}(-x)^{k} \frac{w_{1}^{m_{1}}}{m_{1} !} \frac{w_{2}^{m_{2}}}{m_{2} !} \frac{t^{n}}{(n-k) !} \\
& =\sum_{m_{1}, m_{2}, n, k=0}^{\infty} f\left(n+m_{1}+m_{2}+k\right) \frac{(-x t)^{k}}{k !} A_{m_{1}+m_{2}+n+k, m_{2}+k} \frac{w_{1}^{m_{1}}}{m_{1} !} \frac{w_{2}^{m_{2}}}{m_{2} !} \frac{t^{n}}{n !} .
\end{aligned}
$$

Let $m_{1} \rightarrow m_{1}-n$,

$$
\begin{aligned}
\Psi(x) & =\sum_{m_{1}, m_{2}, k=0}^{\infty} f\left(m_{1}+m_{2}+k\right) \frac{(-x t)^{k}}{k !} A_{m_{1}+m_{2}+k, m_{2}+k}\left(\sum_{n=0}^{m_{1}} \frac{w_{1}^{m_{1}-n} t^{n}}{\left(m_{1}-n\right) ! n !}\right) \frac{w_{2}^{m_{2}}}{m_{2} !} \\
& =\sum_{m_{1}, m_{2}, k=0}^{\infty} f\left(m_{1}+m_{2}+k\right) \frac{(-x t)^{k}}{k !} A_{m_{1}+m_{2}+k, m_{2}+k}\left(\frac{\sum_{n=0}^{m_{1}}\left(\begin{array}{c}
m_{1} \\
n
\end{array}\right) w_{1}^{m_{1}-n} t^{n}}{m_{1} !}\right) \frac{w_{2}^{m_{2}}}{m_{2} !} \\
& =\sum_{m_{1}, m_{2}, k=0}^{\infty} f\left(m_{1}+m_{2}+k\right) A_{m_{1}+m_{2}+k, m_{2}+k} \frac{\left(w_{1}+t\right)^{m_{1}}}{m_{1} !} \frac{w_{2}^{m_{2}}}{m_{2} !} \frac{(-x t)^{k}}{k !} .
\end{aligned}
$$

Let $m_{2} \rightarrow m_{2}-k$,

$$
\begin{aligned}
\Psi(x) & =\sum_{m_{1}, m_{2}=0}^{\infty} f\left(m_{1}+m_{2}\right) A_{m_{1}+m_{2}, m_{2}} \frac{\left(w_{1}+t\right)^{m_{1}}}{m_{1} !} \sum_{k=0}^{m_{2}} \frac{w_{2}^{m_{2}-k}(-x t)^{k}}{\left(m_{2}-k\right) ! k !} \\
& =\sum_{m_{1}, m_{2}=0}^{\infty} f\left(m_{1}+m_{2}\right) A_{m_{1}+m_{2}, m_{2}} \frac{\left(w_{1}+t\right)^{m_{1}}}{m_{1} !} \frac{\left(w_{2}+(-x t)\right)^{m_{2}}}{m_{2} !} .
\end{aligned}
$$

Remark 2.5 Choosing $A_{m, n}=(-\alpha-m)_{n}\left(m, n \in \mathbb{N}_{0}\right)$ and $x \rightarrow-\frac{1}{x}$, then by Theorem 2.4, we get

$$
\begin{aligned}
& \sum_{m_{1}, m_{2}, n=0}^{\infty} f\left(n+m_{1}+m_{2}\right)(-1)^{m_{2}}\left(\alpha+m_{1}+n+1\right)_{m_{2}} L_{n}^{\left(\alpha+m_{1}\right)}(x) \frac{w_{1}^{m_{1}}}{m_{1} !} \frac{w_{2}^{m_{2}}}{m_{2} !}\left(-\frac{t}{x}\right)^{n} \\
& =\sum_{m_{1}, m_{2}=0}^{\infty} f\left(m_{1}+m_{2}\right)\left(-\alpha-m_{1}-m_{2}\right)_{m_{2}} \frac{\left(w_{1}+t\right)^{m_{1}}}{m_{1} !} \frac{\left(w_{2}+\left(\frac{t}{x}\right)\right)^{m_{2}}}{m_{2} !} .
\end{aligned}
$$

If we set $w_{2}=-\frac{t}{x}$ and $f=1$ into the above equation, then we have

$$
\begin{aligned}
& \sum_{m_{1}, m_{2}, n=0}^{\infty}(-1)^{m_{2}}\left(\alpha+m_{1}+n+1\right)_{m_{2}} L_{n}^{\left(\alpha+m_{1}\right)}(x) \frac{w_{1}^{m_{1}}}{m_{1} !} \frac{\left(-\frac{t}{x}\right)^{n+m_{2}}}{m_{2} !} \\
& \quad=\sum_{m_{1}=0}^{\infty} \frac{\left(w_{1}+t\right)^{m_{1}}}{m_{1} !}=e^{w_{1}+t} .
\end{aligned}
$$


Remark 2.6 Setting

$$
A_{m, n}=\frac{(\alpha+\beta+1)_{2 m}(-\beta-m)_{n}}{(\alpha+\beta+1)_{m}(-\alpha-\beta-2 m)_{n}} \quad\left(m, n \in \mathbb{N}_{0}\right)
$$

and $x \rightarrow \frac{2}{1+x}$ in equation (6), we have

$$
\begin{aligned}
& \sum_{m_{1}, m_{2}, n=0}^{\infty} f\left(n+m_{1}+m_{2}\right) \\
& \quad \times \frac{(\alpha+\beta+1)_{2 m_{1}+2 m_{2}+2 n}\left(-\beta-m_{1}-m_{2}-n\right)_{m_{2}}\left(1+\alpha+\beta+2 m_{1}+m_{2}\right)_{n}}{(\alpha+\beta+1)_{m_{1}+m_{2}+n}\left(-\alpha-\beta-2 m_{1}-2 m_{2}-2 n\right)_{m_{2}}\left(1+\alpha+\beta+2 m_{1}+m_{2}\right)_{2 n}} \\
& \quad \times n !\left(\frac{2}{1+x}\right)^{n} P_{n}^{\left(\alpha+m_{1}+m_{2}, \beta+m_{1}\right)}(x) \frac{w_{1}^{m_{1}}}{m_{1} !} \frac{w_{2}^{m_{2}}}{m_{2} !} t^{n} \\
& =\sum_{m_{1}, m_{2}=0}^{\infty} f\left(m_{1}+m_{2}\right) \frac{(\alpha+\beta+1)_{2 m_{1}+2 m_{2}}\left(-\beta-m_{1}-m_{2}\right)_{m_{2}}}{(\alpha+\beta+1)_{m_{1}+m_{2}}\left(-\alpha-\beta-2 m_{1}-2 m_{2}\right)_{m_{2}}} \\
& \quad \times \frac{\left(w_{1}+t\right)^{m_{1}}}{m_{1} !} \frac{\left(w_{2}+\left(-\frac{2}{1+x} t\right)\right)^{m_{2}}}{m_{2} !} .
\end{aligned}
$$

Remark 2.7 If we set $A_{m, n}=(\alpha+m-1)_{n}\left(m, n \in \mathbb{N}_{0}\right)$ and $x \rightarrow-\frac{x}{\beta}$ in (6), then we can write

$$
\begin{aligned}
& \sum_{m_{1}, m_{2}, n=0}^{\infty} f\left(n+m_{1}+m_{2}\right)\left(\alpha+m_{1}+m_{2}+n-1\right)_{m_{2}} y_{n}\left(x, \alpha+m_{1}+2 m_{2}, \beta\right) \frac{w_{1}^{m_{1}}}{m_{1} !} \frac{w_{2}^{m_{2}}}{m_{2} !} \frac{t^{n}}{n !} \\
& =\sum_{m_{1}, m_{2}=0}^{\infty} f\left(m_{1}+m_{2}\right)\left(\alpha+m_{1}+m_{2}-1\right)_{m_{2}} \frac{\left(w_{1}+t\right)^{m_{1}}}{m_{1} !} \frac{\left(w_{2}+\frac{x}{\beta} t\right)^{m_{2}}}{m_{2} !} .
\end{aligned}
$$

If we set $f=1$ and $w_{2}=-\frac{x}{\beta} t$, then

$$
\begin{aligned}
& \sum_{m_{1}, m_{2}, n=0}^{\infty}\left(\alpha+m_{1}+m_{2}+n-1\right)_{m_{2}} y_{n}\left(x, \alpha+m_{1}+2 m_{2}, \beta\right) \frac{w_{1}^{m_{1}}}{m_{1} !} \frac{\left(-\frac{x}{\beta} t\right)^{m_{2}}}{m_{2} !} \frac{t^{n}}{n !} \\
& =\sum_{m_{1}=0}^{\infty} \frac{\left(w_{1}+t\right)^{m_{1}}}{m_{1} !}=e^{w_{1}+t} .
\end{aligned}
$$

Remark 2.8 If we consider Remarks 2.5 and 2.7, we get the following relation between Laguerre polynomials $L_{n}^{(\alpha)}(x)$ and Bessel polynomials $y_{n}(x, \alpha, \beta)$ :

$$
\begin{aligned}
& \sum_{m_{1}, m_{2}, n=0}^{\infty}(-1)^{m_{2}}\left(\alpha+m_{1}+n+1\right)_{m_{2}} L_{n}^{\left(\alpha+m_{1}\right)}(x) \frac{w_{1}^{m_{1}}}{m_{1} !} \frac{\left(-\frac{t}{x}\right)^{n+m_{2}}}{m_{2} !} \\
& \quad=\sum_{m_{1}, m_{2}, n=0}^{\infty}\left(\alpha+m_{1}+m_{2}+n-1\right)_{m_{2}} y_{n}\left(x, \alpha+m_{1}+2 m_{2}, \beta\right) \frac{w_{1}^{m_{1}}}{m_{1} !} \frac{\left(-\frac{x}{\beta} t\right)^{m_{2}}}{m_{2} !} \frac{t^{n}}{n !} .
\end{aligned}
$$




\section{Two-parameter two-variable Srivastava polynomials}

In this section we introduce the following two-parameter family of bivariate polynomials:

$$
S_{n}^{m_{1}, m_{2}}(x, y):=\sum_{k=0}^{n} A_{m_{1}+m_{2}+n, m_{2}+k} \frac{x^{k}}{k !} \frac{y^{n-k}}{(n-k) !} \quad\left(m_{1}, m_{2}, n, k \in \mathbb{N}_{0}\right),
$$

where $\left\{A_{n, k}\right\}$ is a bounded double sequence of real or complex numbers. Note that in the particular case these polynomials include the Lagrange polynomials.

Remark 3.1 Choosing $A_{m, n}=(\alpha)_{m-n}(\beta)_{n}\left(m, n \in \mathbb{N}_{0}\right)$ in (7), we have

$$
S_{n}^{m_{1}, m_{2}}(x, y)=(\alpha)_{m_{1}}(\beta)_{m_{2}} g_{n}^{\left(\alpha+m_{1}, \beta+m_{2}\right)}(x, y)
$$

where $g_{n}^{(\alpha, \beta)}(x, y)$ are the Lagrange polynomials given by

$$
g_{n}^{(\alpha, \beta)}(x, y)=\sum_{k=0}^{n} \frac{(\alpha)_{n-k}(\beta)_{k}}{(n-k) ! k !} x^{k} y^{n-k}
$$

Using similar techniques as in the proof of Theorem 2.4, we get the following theorem.

Theorem 3.2 Let $\{f(n)\}_{n=0}^{\infty}$ be a bounded sequence of complex numbers. Then

$$
\begin{aligned}
& \sum_{m_{1}, m_{2}, n=0}^{\infty} f\left(n+m_{1}+m_{2}\right) S_{n}^{m_{1}, m_{2}}(x, y) \frac{w_{1}^{m_{1}}}{m_{1} !} \frac{w_{2}^{m_{2}}}{m_{2} !} t^{n} \\
& =\sum_{m_{1}, m_{2}=0}^{\infty} f\left(m_{1}+m_{2}\right) A_{m_{1}+m_{2}, m_{2}} \frac{\left(w_{1}+y t\right)^{m_{1}}}{m_{1} !} \frac{\left(w_{2}+x t\right)^{m_{2}}}{m_{2} !}
\end{aligned}
$$

provided each member of the series identity (8) exists.

Remark 3.3 If we set $A_{m, n}=(\alpha)_{m-n}(\beta)_{n}\left(m, n \in \mathbb{N}_{0}\right)$ in (8), we have

$$
\begin{aligned}
& \sum_{m_{1}, m_{2}, n=0}^{\infty} f\left(n+m_{1}+m_{2}\right)(\alpha)_{m_{1}}(\beta)_{m_{2}} g_{n}^{\left(\alpha+m_{1}, \beta+m_{2}\right)}(x, y) \frac{w_{1}^{m_{1}}}{m_{1} !} \frac{w_{2}^{m_{2}}}{m_{2} !} t^{n} \\
& =\sum_{m_{1}, m_{2}=0}^{\infty} f\left(m_{1}+m_{2}\right)(\alpha)_{m_{1}}(\beta)_{m_{2}} \frac{\left(w_{1}+y t\right)^{m_{1}}}{m_{1} !} \frac{\left(w_{2}+x t\right)^{m_{2}}}{m_{2} !} .
\end{aligned}
$$

Choosing $f=1$ gives

$$
\begin{aligned}
& \sum_{m_{1}, m_{2}, n=0}^{\infty}(\alpha)_{m_{1}}(\beta)_{m_{2}} g_{n}^{\left(\alpha+m_{1}, \beta+m_{2}\right)}(x, y) \frac{w_{1}^{m_{1}}}{m_{1} !} \frac{w_{2}^{m_{2}}}{m_{2} !} t^{n} \\
& =\left(1-w_{1}-y t\right)^{-\alpha}\left(1-w_{2}-x t\right)^{-\beta} .
\end{aligned}
$$

Furthermore, since we have the relation between $P_{n}^{(\alpha, \beta)}(x, y)$ Jacobi polynomials and Lagrange polynomials [9] as

$$
g_{n}^{(\alpha, \beta)}(x, y)=(y-x)^{n} P_{n}^{(-\alpha-n,-\beta-n)}\left(\frac{x+y}{x-y}\right),
$$


we get the following generating relation for Jacobi polynomials $P_{n}^{(\alpha, \beta)}(x, y)$ :

$$
\begin{aligned}
& \sum_{m_{1}, m_{2}, n=0}^{\infty}(\alpha)_{m_{1}}(\beta)_{m_{2}}(y-x)^{n} P_{n}^{\left(-\alpha-m_{1}-n,-\beta-m_{2}-n\right)}\left(\frac{x+y}{x-y}\right) \frac{w_{1}^{m_{1}}}{m_{1} !} \frac{w_{2}^{m_{2}}}{m_{2} !} t^{n} \\
& =\left(1-w_{1}-y t\right)^{-\alpha}\left(1-w_{2}-x t\right)^{-\beta} .
\end{aligned}
$$

\section{Two-parameter three-variable Srivastava polynomials}

In this section we define two-parameter three-variable Srivastava polynomials as follows:

$$
\begin{aligned}
S_{n}^{m_{1}, m_{2}, M}(x, y, z) & :=\sum_{k=0}^{n} \sum_{l=0}^{\left[\frac{k}{M}\right]} A_{m_{1}+m_{2}+n, m_{2}+k, l} \frac{x^{l}}{l !} \frac{y^{k-M l}}{(k-M l) !} \frac{z^{n-k}}{(n-k) !} \\
\left(m_{1}, m_{2}, n, k, l\right. & \left.\in \mathbb{N}_{0}, M \in \mathbb{N}\right),
\end{aligned}
$$

where $\left\{A_{n, k, l}\right\}_{n, k=0}^{\infty}$ is a bounded triple sequence of real or complex numbers.

Using similar techniques as in the proof of Theorem 2.4, we get the following theorem.

Theorem 4.1 Let $\{f(n)\}_{n=0}^{\infty}$ be a bounded sequence of complex numbers. Then

$$
\begin{aligned}
& \sum_{m_{1}, m_{2}, n=0}^{\infty} f\left(n+m_{1}+m_{2}\right) S_{n}^{m_{1}, m_{2}, M}(x, y, z) \frac{w_{1}^{m_{1}}}{m_{1} !} \frac{w_{2}^{m_{2}}}{m_{2} !} t^{n} \\
& =\sum_{m_{1}, m_{2}, l=0}^{\infty} f\left(m_{1}+m_{2}+M l\right) A_{m_{1}+m_{2}+M l, m_{2}+M l, l} \frac{\left(x t^{M}\right)^{l}}{l !} \frac{\left(w_{1}+z t\right)^{m_{1}}}{m_{1} !} \frac{\left(w_{2}+y t\right)^{m_{2}}}{m_{2} !},
\end{aligned}
$$

provided each member of the series identity (10) exists.

Theorem 4.2 Let $\{f(n)\}_{n=0}^{\infty}$ be a bounded sequence of complex numbers, and let $S_{n}^{m_{1}, m_{2}, M}(x$, $y, z)$ be defined by (9). Suppose also that two-parameter two-variable polynomials $P_{m_{1}, m_{2}}^{M}(x$, y) are defined by

$$
P_{m_{1}, m_{2}}^{M}(x, y)=\sum_{l=0}^{\left[\frac{m_{2}}{M}\right]} A_{m_{1}+m_{2}, m_{2}, l} \frac{x^{m_{2}-M l}}{\left(m_{2}-M l\right) !} \frac{y^{l}}{l !} .
$$

Then the family of two-sided linear generating relations holds true between the twoparameter three-variable Srivastava polynomials $S_{n}^{m_{1}, m_{2}, M}(x, y, z)$ and $P_{m_{1}, m_{2}}^{M}(x, y)$ :

$$
\begin{aligned}
& \sum_{m_{1}, m_{2}, n=0}^{\infty} f\left(n+m_{1}+m_{2}\right) S_{n}^{m_{1}, m_{2}, M}(x, y, z) \frac{w_{1}^{m_{1}}}{m_{1} !} \frac{w_{2}^{m_{2}}}{m_{2} !} t^{n} \\
& =\sum_{m_{1}, m_{2}=0}^{\infty} f\left(m_{1}+m_{2}\right) \frac{\left(w_{1}+z t\right)^{m_{1}}}{m_{1} !} P_{m_{1}, m_{2}}^{M}\left(w_{2}+y t, x t^{M}\right) .
\end{aligned}
$$

Suitable choices of $A_{n, k, l}$ in equations (9) and (11) give some known polynomials.

Remark 4.3 Choosing $M=2$ and $A_{m, n, k}=(\alpha)_{m-n}(\gamma)_{n-2 k}(\beta)_{k}\left(m, n \in \mathbb{N}_{0}\right)$ in (9), we get

$$
S_{n}^{m_{1}, m_{2}, 2}(x, y, z)=(\alpha)_{m_{1}}(\gamma)_{m_{2}} u_{n}^{\left(\alpha+m_{1}, \beta, \gamma+m_{2}\right)}(z, x, y),
$$


where $u_{n}^{(\alpha, \beta, \gamma)}(x, y, z)$ is the polynomial given by

$$
u_{n}^{(\alpha, \beta, \gamma)}(x, y, z)=\sum_{k=0}^{n} \sum_{l=0}^{\left[\frac{k}{2}\right]}(\beta)_{l}(\gamma)_{k-2 l}(\alpha)_{n-k} \frac{y^{l}}{l !} \frac{x^{n-k}}{(n-k) !} \frac{z^{k-2 l}}{(k-2 l) !} .
$$

Now, by setting $M=1$ and $A_{m, n, k}=(\alpha)_{k}(\beta)_{n-k}(\gamma)_{m-n}\left(m, n \in \mathbb{N}_{0}\right)$ in the definition (9), we obtain

$$
S_{n}^{m_{1}, m_{2}, 1}(x, y, z)=(\gamma)_{m_{1}}(\beta)_{m_{2}} g_{n}^{\left(\alpha, \beta+m_{2}, \gamma+m_{1}\right)}(x, y, z),
$$

where $g_{n}^{(\alpha, \beta, \gamma)}(x, y, z)$ are the Lagrange polynomials given by

$$
g_{n}^{(\alpha, \beta, \gamma)}(x, y, z)=\sum_{k=0}^{n} \sum_{l=0}^{k}(\alpha)_{l}(\beta)_{k-l}(\gamma)_{n-k} \frac{x^{l}}{l !} \frac{y^{k-l}}{(k-l) !} \frac{z^{n-k}}{(n-k) !} .
$$

Remark 4.4 If we set $M=2$ and $A_{m, n, k}=(\alpha)_{m-n}(\gamma)_{n-2 k}(\beta)_{k}\left(m, n \in \mathbb{N}_{0}\right)$ in (11), then

$$
P_{m_{1}, m_{2}}^{2}(x, y)=(\alpha)_{m_{1}} h_{m_{2}}^{(\gamma, \beta)}(x, y),
$$

where $h_{m_{2}}^{(\gamma, \beta)}(x, y)$ denotes the Lagrange-Hermite polynomials given explicitly by

$$
h_{m_{2}}^{(\gamma, \beta)}(x, y)=\sum_{l=0}^{\left[\frac{m_{2}}{2}\right]}(\gamma)_{m_{2}-2 l}(\beta)_{l} \frac{x^{m_{2}-2 l}}{\left(m_{2}-2 l\right) !} \frac{y^{l}}{l !} .
$$

Furthermore, choosing $M=1$ and $A_{m, n, k}=(\alpha)_{k}(\beta)_{n-k}(\gamma)_{m-n}\left(m, n \in \mathbb{N}_{0}\right)$ in the definition (11), we have

$$
P_{m_{1}, m_{2}}^{1}(x, y)=(\gamma)_{m_{1}} g_{m_{2}}^{(\beta, \alpha)}(x, y),
$$

where $g_{m_{2}}^{(\alpha, \beta)}(x, y)$ are the Lagrange polynomials given by

$$
g_{m_{2}}^{(\alpha, \beta)}(x, y)=\sum_{l=0}^{m_{2}}(\alpha)_{m_{2}-l}(\beta)_{l} \frac{x^{m_{2}-l}}{\left(m_{2}-l\right) !} \frac{y^{l}}{l !} .
$$

Remark 4.5 If we set $w_{1} \rightarrow-z t$ and $w_{2} \rightarrow-y t$ in Theorem 4.1, then we get

$$
\begin{aligned}
& \sum_{m_{1}, m_{2}, n=0}^{\infty} f\left(n+m_{1}+m_{2}\right) S_{n}^{m_{1}, m_{2}, M}(x, y, z) \frac{(-z t)^{m_{1}}}{m_{1} !} \frac{(-y t)^{m_{2}}}{m_{2} !} t^{n} \\
& =\sum_{l=0}^{\infty} f(M l) A_{M l, M l, l} \frac{\left(x t^{M}\right)^{l}}{l !} .
\end{aligned}
$$

Now, if we set $f=1, M=2$ and $A_{m, n, k}=(\alpha)_{m-n}(\gamma)_{n-2 k}(\beta)_{k}\left(m, n \in \mathbb{N}_{0}\right)$ in (13), then

$$
\sum_{m_{1}, m_{2}, n=0}^{\infty}(\alpha)_{m_{1}}(\gamma)_{m_{2}} u_{n}^{\left(\alpha+m_{1}, \beta, \gamma+m_{2}\right)}(z, x, y) \frac{(-z t)^{m_{1}}}{m_{1} !} \frac{(-y t)^{m_{2}}}{m_{2} !} t^{n}=\left(1-x t^{2}\right)^{-\beta}
$$


Furthermore, if we set $M=2$ and $A_{m, n, k}=(\alpha)_{m-n}(\gamma)_{n-2 k}(\beta)_{k}\left(m, n \in \mathbb{N}_{0}\right)$ in (12), then

$$
\begin{aligned}
& \sum_{m_{1}, m_{2}, n=0}^{\infty} f\left(n+m_{1}+m_{2}\right)(\alpha)_{m_{1}}(\gamma)_{m_{2}} u_{n}^{\left(\alpha+m_{1}, \beta, \gamma+m_{2}\right)}(z, x, y) \frac{w_{1}^{m_{1}}}{m_{1} !} \frac{w_{2}^{m_{2}}}{m_{2} !} t^{n} \\
& =\sum_{m_{1}, m_{2}=0}^{\infty} f\left(m_{1}+m_{2}\right) \frac{\left(w_{1}+z t\right)^{m_{1}}}{m_{1} !}(\alpha)_{m_{1}} h_{m_{2}}^{(\gamma, \beta)}\left(w_{2}+y t, x t^{2}\right) .
\end{aligned}
$$

\section{Competing interests}

The authors declare that they have no competing interests.

\section{Authors' contributions}

Both authors completed the paper together. Both authors read and approved the final manuscript.

\section{Author details}

${ }^{1}$ Faculty of Engineering, Cyprus International University, Mersin 10, Nicosia, Turkey. ${ }^{2}$ Department of Mathematics, Faculty of Arts and Sciences, Eastern Mediterranean University, Mersin 10, Gazimagusa, Turkey.

\section{Acknowledgements}

Dedicated to Professor Hari M Srivastava

We would like to thank the referees for their valuable comments.

Received: 12 December 2012 Accepted: 6 March 2013 Published: 29 March 2013

\section{References}

1. Srivastava, HM: A contour integral involving Fox's H-function. Indian J. Math. 14, 1-6 (1972)

2. González, B, Matera, J, Srivastava, HM: Some $q$-generating functions and associated generalized hypergeometric polynomials. Math. Comput. Model. 34, 133-175 (2001)

3. Altın, A, Erkuş, E, Özarslan, MA: Families of linear generating functions for polynomials in two variables. Integral Transforms Spec. Funct. 17(5), 315-320 (2006)

4. Srivastava, HM, Özarslan, MA, Kaanoglu, C: Some families of generating functions for a certain class of three-variable polynomials. Integral Transforms Spec. Funct. 21(12), 885-896 (2010)

5. Kaanoglu, C, Özarslan, MA: New families of generating functions for certain class of three-variable polynomials. Appl. Math. Comput. 218, 836-842 (2011)

6. Kaanoglu, C, Özarslan, MA: Two-sided generating functions for certain class of $r$-variable polynomials. Math. Comput. Model. 54, 625-631 (2011)

7. Özarslan, MA: Some families of generating functions for the extended Srivastava polynomials. Appl. Math. Comput. 218, 959-964 (2011)

8. Liu, S-J, Chyan, C-J, Lu, H-C, Srivastava, HM: Multiple integral representations for some families of hypergeometric and other polynomials. Math. Comput. Model. 54, 1420-1427 (2011)

9. Srivastava, HM, Manocha, HL: A Treatise on Generating Functions. Halsted, New York (1984)

doi:10.1186/1687-1847-2013-81

Cite this article as: Kaanoglu and Özarslan: Two-parameter Srivastava polynomials and several series identities. Advances in Difference Equations 2013 2013:81.

\section{Submit your manuscript to a SpringerOpen ${ }^{\circ}$ journal and benefit from:}

- Convenient online submission

- Rigorous peer review

Immediate publication on acceptance

- Open access: articles freely available online

- High visibility within the field

- Retaining the copyright to your article 